(2) Open Access Full Text Article

\title{
Improving engagement in mental health treatment for home meal recipients with depression
}

This article was published in the following Dove Press journal:

Clinical Interventions in Aging

26 September 2013

Number of times this article has been viewed

Jo Anne Sirey

Alexandra Greenfield

Alyssa DePasquale

Nathalie Weiss

Patricia Marino

George S Alexopoulos

Martha L Bruce

Department of Psychiatry,

Weill Cornell Medical College,

White Plains, NY, USA
Correspondence: Jo Anne Sirey, PhD Department of Psychiatry, Weill Cornell Medical College,

2I Bloomingdale Rd, White Plains,

NY 10605, USA

Tel + I 9149974333

Fax + I 9149978650

Email jsirey@med.cornell.edu
Background: Staff who provide support services to older adults are in a unique position to detect depression and offer a referral for mental health treatment. Yet integrating mental health screening and recommendations into aging services requires staff learn new skills to integrate mental health and overcome client barriers to accepting mental health referrals. This paper describes client rates of depression and a novel engagement intervention (Open Door) for homebound older adults who are eligible for home delivered meals and screened for depression by in-home aging service programs.

Methods: Homebound older adults receiving meal service who endorsed depressive symptoms were interviewed to assess depression severity and rates of suicidal ideation. Open Door is a brief psychosocial intervention to improve engagement in mental health treatment by collaboratively addressing the individual level barriers to care. The intervention targets stigma, misconceptions about depression, and fears about treatment, and is designed to fit within the roles and responsibilities of aging service staff.

Results: Among 137 meal recipients who had symptoms when screened for depression as part of routine home meal service assessments, half (51\%) had Major Depressive Disorder and 13\% met criteria for minor depression on the SCID. Suicidal ideation was reported by $29 \%$ of the sample, with the highest rates of suicidal ideation (47\%) among the subgroup of individuals with Major Depressive Disorder.

Conclusion: Individuals who endorse depressive symptoms during screening are likely to have clinically significant depression and need mental health treatment. The Open Door intervention offers a strategy to overcome barriers to mental health treatment engagement and to improve the odds of quality care for depression.

Keywords: depression, access to care, mental health intervention, engagement

\section{Introduction}

Initiatives implemented by aging service agencies to screen older adults for depression and other mental health or substance abuse problems are an important first step to reduce unmet need for mental health services. ${ }^{1,2}$ However, the success of screening in improving the link to mental health services rests largely on a successful referral process. To accept a referral and engage in mental health treatment may entail striking a balance between perceived need, the social costs of mental health care (eg, stigma, personal rejection), negative attitudes towards depression and its treatment, and overcoming tangible access obstacles. ${ }^{3,4}$ Many depressed older adults identified in aging services are willing to use mental health treatment, ${ }^{5}$ but making an effective referral that results in treatment engagement is a skill. 
In older adults, depression is associated with the risk of deterioration of cognition and medical status, ${ }^{6}$ increased disability, ${ }^{7,8}$ increased risk of falls, ${ }^{9}$ and compromised quality of life. ${ }^{7,10}$ Mental illness among older adults is also associated with excess utilization of health care, increased placement in nursing homes, greater burden to medical care providers, and higher annual health care costs. ${ }^{11-15}$ Depression in particular worsens the outcomes of many medical disorders and increases the risk for falls, ${ }^{16}$ suicide $^{17}$ and non-suicide mortality. ${ }^{18-21}$ Mental health and behavioral disorders are the second leading cause of disability worldwide among non-communicable diseases. ${ }^{22}$ Of these disorders, unipolar depressive disorders have the highest burden in disabilityadjusted life years, and it is estimated that this burden will continue to increase. Rates of depression are increased in samples of community-dwelling older persons with greater medical illness or disability. ${ }^{23}$

The level of unmet mental health need is high in older adults. A recent analysis of older adults in the National Comorbidity Study Replication sample found that $70 \%$ of older adults with mood and anxiety disorders did not receive mental health treatment. ${ }^{24}$ In a 9-year community follow-up study of older adults with depressive symptoms, 43\%-69\% did not receive treatment for depression during any 18-month interval. In addition, $31 \%$ of the 121 participants with persistent depression did not receive mental health treatment during the study period. ${ }^{25}$

Home-based agency services offer a unique opportunity to address unmet mental health needs in a very high-risk population. The Elderly Nutrition Program is funded by the US Department of Health and Human Services to support older individuals' tenure in the home through meal provision to homebound elders; these meals sustain adequate dietary patterns and improve nutritional intake. ${ }^{26}$ The home meal program serves the fastest growing older adult population in the US, ie, persons aged 85 years and older. ${ }^{27}$ In a community partnership study to examine mental health need in the home meal program, $12 \%$ of clients receiving meals reported clinically significant depression (Patient Health Questionnaire-9 score $>9$ ), with an additional $15 \%$ reporting mild depressive symptoms, and $13 \%$ of clients screened endorsed thoughts of death and dying. ${ }^{28} \mathrm{~A}$ recent assessment of mental health need using a research diagnostic interview reported that $27 \%$ of aging service clients receiving case management met criteria for a current major depressive episode. ${ }^{29}$

In this paper, we describe the levels of depression and suicidal ideation among older persons participating in the home meal program and an intervention to improve engagement in mental health treatment delivered in aging services. We hypothesized that diagnostic interviews would yield significant rates of major depression among clients participating in the home meal program who endorse symptoms when screened for depression as part of routine home meal service assessments. We targeted this group of older adults because home meal program recipients require meal service to maintain their independence and untreated depression can increase their potential disability and decline. Regular assessments for home meal program eligibility provide an opportunity to integrate routine depression screening followed by an intervention to link depressed older adults to mental health treatment.

\section{Materials and methods Target population}

The US Department of Health and Human Services, Administration for Community Living (Administration on Aging) authorizes provision of meals to individuals who are homebound, with nearly 149 million home-delivered meals provided to more than 880,000 homebound individuals across the US in $2009 .{ }^{30}$ Compared with the overall older adult US population (age $\geq 60$ years), these home meal recipients are more likely to be older, poor, black, and living alone, to be in poor health, have greater difficulty performing everyday tasks, and to be at high nutritional risk. ${ }^{31}$ To be eligible for a home-delivered meals program, an older adult must be "confined" due to a condition, illness, or injury that restricts the ability of the individual to leave the home except with some type of assistance.

\section{Sample and recruitment}

The sample is part of a larger study (National Institute of Mental Health R01 MH079265) to test the effectiveness of the Open Door intervention. Participants are adults aged 60 years or older who are eligible for home meal service in Westchester County and Bronx, NY, USA, and who endorse symptoms when screened for depression using the Patient Health Questionnaire-2 or Patient Health Questionnaire-932 as part of routine home assessment (see Figure 1). Once an eligible subject is identified through routine depression screening, verbal consent is obtained for research staff to contact the older adult and describe the study. If the subject chooses to participate, a counselor makes an in-home visit to obtain informed consent and conduct a baseline assessment. Exclusion criteria, evaluated by research staff, include 


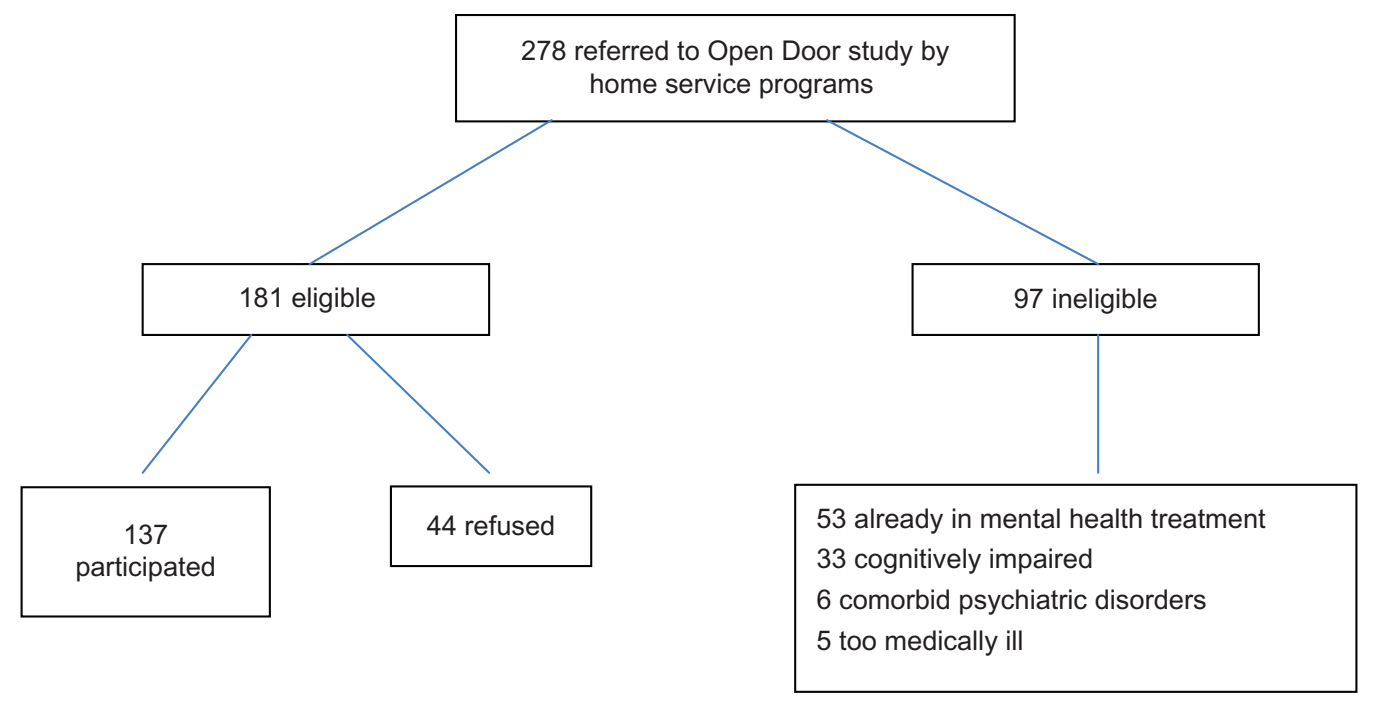

Figure I Recruitment for Open Door study.

the presence of significant substance abuse or psychotic disorder, active suicidal ideation requiring immediate attention, significant cognitive impairment, aphasia interfering with communication, inability to speak English, or already participating in mental health treatment (either antidepressant medication or psychotherapy). The study was approved by the Weill Cornell institutional review board (Protocol 0707009247).

During baseline assessment, the level of mental health need (depression diagnosis, depression severity, and presence of suicidal ideation), functioning, and attitudes towards depression and its treatment are evaluated. The Structured Clinical Interview for Diagnostic and Statistical Manual of Mental Disorders, Fourth Edition (DSM-IV) Axis I disorders ${ }^{33}$ is used to determine the current diagnosis, and severity ratings are conducted using the Montgomery Asberg Depression Rating Scale (MADRS).$^{34}$ The inter-rater consistency is $94 \%$ for the MADRS between all study raters conducting baseline and follow-up assessments.

Individuals who endorse active or passive suicidal ideation are evaluated using the Suicide Risk Assessment ${ }^{35}$ to determine the suicide risk level and an appropriate course of action. The Suicide Risk Assessment questions elicit information about suicidal thoughts, presence of a plan, wish to die, and reasons for living. Mild risk is defined as thoughts about suicide, a strong wish to die, or a past history of attempted suicide. For mild risk, the recommendation is that suicidal thoughts be discussed as part of the mental health treatment being recommended. If the individual has attempted suicide in the past month, has had a previous attempt requiring medical attention, endorses a strong wish to die, has considered a method in the past month, or has thoughts of committing suicide but has not considered a method, then the client is considered intermediate risk. Clients with intermediate risk are reviewed with a supervisor and a contract is created, and with the client's permission, the client's primary care clinician or a family member is notified. If there is suicidal intent with nothing preventing the individual from attempting suicide or there is questionable impulse control, then the client is considered high-risk. These clients require immediate intervention (eg, crisis team, police, or hospitalization). These risk assessment and suicide risk review procedures have been used in multiple protocols for community-based research..$^{35,36}$

Ability to complete instrumental activities of daily living and the number of medical conditions are assessed using the Multi-level Assessment Inventory. ${ }^{37}$ Cognitive functioning is evaluated using the Mini-Mental Status Examination, ${ }^{38}$ with individuals who score less than 24 excluded from participation. The level of nutritional risk is evaluated using a standard questionnaire administered by all home meal programs in New York State. ${ }^{39}$ This questionnaire yields a score from 0 to 21, and an individual score of 6 or greater is considered at high nutritional risk. Chronic pain is assessed using self-report questions to evaluate the presence of painful sensations during the preceding 6 months. ${ }^{40}$ Participants are also asked if they have fallen during the previous 6 months, and if so, whether they sustained any injuries from a fall and if they saw a physician for these injuries. Prior use of mental health treatment is documented using the Cornell Services Index. ${ }^{41}$ 


\section{Engagement intervention}

The Open Door intervention is a brief, individualized intervention to identify and address barriers to engagement in mental health treatment among older persons whose depression is detected in aging service settings. The premise of the intervention is that by collaboratively engaging the older adult in the process of seeking mental health treatment, the intervention both creates an engagement plan that is personalized and models the collaborative process of quality mental health treatment. ${ }^{42} \mathrm{~A}$ referral is the first step to engagement, but often referrals are not accepted. The Open Door intervention is conducted during three face-to-face, 30-minute intervention meetings in the client's home with one telephone follow-up. The Open Door intervention was taught during a two-day training provided by the principal study investigator (JAS) with weekly supervision provided for the first 6 months followed by monthly supervision thereafter.

There are five steps to the Open Door intervention: recommend referral, conduct a barriers assessment, define a personal goal that could be achieved with care, provide education about depression treatment options, and finally, address the barriers to accessing care. The Open Door counselor serves a similar function as the patient navigator whose role in a hospital setting is to improve access to cancer screening and treatment. ${ }^{43,44}$ The Open Door intervention is different from other engagement interventions in two ways. First, the barriers assessed as part of the intervention are empirically defined from research identifying health beliefs and attitudes that predict poor treatment participation outcomes, such as not initiating care, ${ }^{45}$ dropping out, ${ }^{46}$ or not following a medication regimen. ${ }^{47}$ Second, the individualized assessment of barriers goes beyond rational decision-making to elicit the beliefs and concerns, including irrational ideas, that may be underlying the reluctance to seek mental health treatment. In some instances, the intervention allows the client to articulate the fears that s/he may be self-conscious about admitting, but have become the basis for not seeking mental health treatment.

During the Open Door intervention meetings, the counselor uses techniques drawn from motivational interviewing to help mobilize an individual's intrinsic motivation to seek help. ${ }^{48}$ The client and counselor use problem-solving techniques to brainstorm about solutions to barriers, weigh the options, and create a specific plan to seek mental health treatment. Sample intervention strategies are shown in Table 1. The client's treatment modality and setting preferences are assessed using a scripted presentation of the available treatment options (eg, primary care physician, mental health provider, research protocol). By participating in this process, the Open Door intervention addresses not only the referral, but also the first steps of the treatment process.

Prior to the current study, a small feasibility pilot was conducted with the Department of Senior Programs and Services, which had documented a 22\% (18/117) acceptance rate of referrals to a mental health resource using their usual referral procedures. In this feasibility pilot, home meal program participants who screened positive for depression on the Patient Health Questionnaire-9 were referred to a mental health resource using the Open Door intervention. Of the 29 participants referred, $20(62 \%)$ accepted a referral to mental health treatment and scheduled a first appointment to be seen by a clinician. This feasibility study supported

Table I Examples of Open Door intervention

\begin{tabular}{|c|c|c|c|}
\hline Psychologic barrier & Open Door intervention activity & $\begin{array}{l}\text { Source of technique } \\
\text { (PST, MI, or PE) }\end{array}$ & Outcome \\
\hline Personal stigma concern: & Validate concern (stigma is real!) & MI - reflective listening and empathy & Support \\
\hline "My neighbor will not include & Define disclosure options & PST - brainstorming & More hope \\
\hline me if she thinks I'm crazy." & Emphasize personal choice & $\mathrm{MI}$ - collaboration & Less helplessness \\
\hline & Review pros and cons of each option & PST - identify pros and cons and compare & Action plan \\
\hline Treatment efficacy concerns: & Identify hopeless as symptoms & PE- - education about depression & Increase in knowledge \\
\hline “What's talking going to do? & of depression & PST - identify a goal & Increased motivation \\
\hline Nothing can change." & $\begin{array}{l}\text { Identify what she wishes to change } \\
\text { Link goal with treatment outcome }\end{array}$ & $\begin{array}{l}\text { PE - review psychotherapy efficacy data } \\
\text { and discuss the process of seeking care }\end{array}$ & Engagement \\
\hline $\begin{array}{l}\text { Attribution of depression symptoms: } \\
\text { "It's the diabetes and my age }\end{array}$ & $\begin{array}{l}\text { Validate overlap of medical and } \\
\text { psychologic symptoms }\end{array}$ & $\begin{array}{l}\text { PE - depression symptom and medical } \\
\text { symptom overlap }\end{array}$ & $\begin{array}{l}\text { Increased knowledge } \\
\text { Increased perceived }\end{array}$ \\
\hline that cause my troubles" & $\begin{array}{l}\text { Describe symptoms of depression } \\
\text { Review myths and potential for } \\
\text { misattribution }\end{array}$ & $\begin{array}{l}\mathrm{PE} \text { - information on depression } \\
\mathrm{PE} \text { - discuss myths and stereotypes }\end{array}$ & need for treatment \\
\hline
\end{tabular}

Abbreviations: PE, psychoeducation; PST, problem-solving therapy; MI, motivational interviewing. 
the delivery of the intervention and the potential to improve mental health referral rates.

\section{Data analysis}

This study analyzed data collected during baseline assessment of the Open Door study. All study data were entered in an Access database and converted to files for analyses using Statistical Package for Social Sciences version 19.0 software. ${ }^{49}$ Descriptive analyses were conducted to characterize the sample. Chi-square and $t$-test analyses were conducted to compare older adults with major depressive disorder and minor or no depression regarding their rates of suicidal ideation, medical conditions, rate of pain, and functioning in instrumental activities of daily living.

\section{Results}

Elderly Nutrition Program staff referred 278 individuals who endorsed symptoms when screened for depression as part of routine home meal service assessments to the Open Door program. All clients who screened positive were referred to determine the level of depression and need for mental health treatment. Of those referred, 97 were ineligible (53 were in mental health treatment, 33 had cognitive impairment, six had other psychiatric disorders, and five were too medically compromised to participate). Of 181 eligible individuals, 24\% (44/181) refused to participate (see Figure 1). The sample consisted of 137 home meal program participants. The demographic characteristics of the sample are described in Table 2. In addition to the home meal program, many participants reported using medical support services in the 3 months prior to their enrollment in the home meal program. One third $(33 \%$, $45 / 137$ ) of the sample had the help of a home health aide and most clients $(85 \%, 116 / 137)$ had been to at least one medical visit. Almost one in five clients $(19 \%, 26 / 137)$ had been hospitalized and $16 \%(22 / 137)$ had made a visit to an emergency room in the previous 3 months.

Within this sample of home meal recipients who screened positive for depression, half of participants assessed (51\%, 70/137) met DSM-IV diagnostic criteria for major depressive disorder and 13\% (18/137) met criteria for minor depression. Older adults who met criteria for major depressive disorder had more medical conditions than those older adults with either minor depression or no depression $(t=-2.78, d f=134$, $P<0.01)$. There were no differences between those with major depressive disorder and those with minor depression or no depression in the number of impairments in instrumental
Table 2 Sample characteristics $(n=137)$

\begin{tabular}{|c|c|c|}
\hline Demographic characteristics & $\begin{array}{l}\text { Number } \\
\text { or range }\end{array}$ & $\begin{array}{l}\text { Percent/mean } \\
\text { (SD) }\end{array}$ \\
\hline Female & 98 & $72 \%$ \\
\hline Age, years & $57-98$ & $78(9.3)$ \\
\hline Hispanic origin & II & $8 \%$ \\
\hline \multicolumn{3}{|l|}{ Race } \\
\hline African American origin & 42 & $31 \%$ \\
\hline Caucasian & 94 & $69 \%$ \\
\hline Other & I & $0.7 \%$ \\
\hline Education, years & $\mathrm{I}-25$ & $12.18(3.7)$ \\
\hline \multicolumn{3}{|l|}{ Marital status } \\
\hline Single & 19 & $14 \%$ \\
\hline Married & 19 & $14 \%$ \\
\hline Once married & 99 & $72 \%$ \\
\hline Live alone & 89 & $65 \%$ \\
\hline \multicolumn{3}{|l|}{ Clinical characteristics } \\
\hline \multicolumn{3}{|l|}{ Depression diagnosis } \\
\hline None & 49 & $36 \%$ \\
\hline Minor & 18 & $13 \%$ \\
\hline Major & 70 & $51 \%$ \\
\hline Endorsed suicidal ideation (\%) & 40 & $29 \%$ \\
\hline Fall in previous 6 months & 50 & $37 \%$ \\
\hline \multicolumn{3}{|l|}{ Service utilization (previous } \\
\hline \multicolumn{3}{|l|}{3 months) } \\
\hline Inpatient hospitalization & 26 & $19 \%$ \\
\hline Emergency room visit & 22 & $16 \%$ \\
\hline Number of medical conditions & $0-14$ & $6.1(2.7)$ \\
\hline Number of current medications & $1-18$ & $6.5(3.3)$ \\
\hline Number of IADL impairments & $0-7$ & $3.4(2.0)$ \\
\hline
\end{tabular}

Abbreviations: IADL, instrumental activities of daily living; SD, standard deviation.

activities of daily living $(t=-0.81, d f=135, P=0.42)$ or those reporting experience of pain during the previous 6 months $\left(\chi^{2}=1.74, d f=1, P=0.19\right)$. Those with major depressive disorder were also more likely to be at nutritional risk. ${ }^{50}$

Almost one third $(29 \%, 40 / 137)$ of participants endorsed suicidal ideation and received the suicide risk assessment. Within the group of participants whose suicide risk was assessed, 23\% (9/40) of clients who screened positive for suicide had intermediate or high risk. Among older adults with major depressive disorder, $47 \%(33 / 70)$ endorsed suicidal ideation and had a risk assessment; with one quarter $(24 \%, 8 / 33)$ of clients with major depressive disorder found to be at intermediate risk and requiring a contract or plan. Suicide risk was higher among participants with major depression than those with minor depression or no depression $\left(\chi^{2}=23.95, d f=2\right.$, $P<0.001)$. Of the older adults without a diagnosis of either minor or major depression, 6\% (3/49) endorsed suicidal ideation. Suicide risk was not associated with race, age, gender, or education. 


\section{Discussion}

This study reinforces the unique opportunity of the aging service network to improve detection of depression and facilitate engagement in mental health treatment to help individuals with significant mental health needs. In this sample of older adults who endorsed symptoms when screened for depression as part of routine home meal service assessments, $51 \%$ met research diagnostic criteria for major depression and an additional $13 \%$ met criteria for minor depression. This group of older adults reported high rates of suicidal ideation, indicating significant potential risk. This is also a group with mental health need that occurs in the context of significant disability, comorbid medical conditions, and social isolation, with the greatest medical burden, pain, and suicidal ideation among older adults with major depression.

The findings of this study echo the emerging research data documenting mental health need among older adults receiving aging services, ${ }^{5,51}$ and the sample is consistent with the characteristics reported nationally for home meal program participants. ${ }^{52}$ While the sampling strategies may vary between studies, the high rate of depression among aging service recipients is consistent with depressive rates reported by other research in this population. ${ }^{5,51}$ However, this study documents high rates of suicidal ideation in this population (29\%), with the highest rate of suicidal ideation reported by adults who met criteria for major depression, where more than half (51\%) of home meal program clients with major depression endorsed suicidal ideation. This finding would suggest that aging service staff should have protocols to address suicide ideation and potential risk as they implement depressive screening and referral programs. Building partnerships between mental health providers and aging services may serve as a bridge for older adults who need mental health treatment. ${ }^{53}$

Home meal program clients also have high levels of disability, medical burden, and vulnerability to falls. Despite limitations in mobility, many home meal program recipients do leave their homes for medical visits, suggesting that there is potential to travel for mental health treatment. The Open Door intervention may offer a strategy to improve engagement in mental health treatment by addressing the need and real barriers to care in this population. The Open Door intervention may improve the perception of acceptability, availability, and accessibility of mental health care by reducing psychologic barriers, providing education about care, and managing the resignation associated with symptoms of depression among community-dwelling depressed elders. Future work should evaluate the acceptability and impact of the Open Door intervention on engagement and attitudes towards mental health treatment, especially stigma.

The limitations of this study include its small sample size and potential bias in recruitment and participation. While the refusal rate was low (24\%) for a community-based study, those individuals who refused may have included older adults most reluctant to seek mental health care, and older adults with the greatest barriers. An additional limitation is the study location. The intervention was delivered in urban and suburban areas where mental health resources may be more available than in other areas. Finally, the Open Door intervention focuses on barriers to care at the individual level but does not mitigate provider or system level barriers that may be insurmountable. However, we believe that addressing psychologic obstacles will improve access and help older adults to anticipate and navigate the often intractable provider and system barriers, such as limited providers. $^{2}$

Despite these limitations, our study findings indicate real and substantial mental health treatment needs among older adults with depressive symptoms identified in the home meal program. The Open Door intervention may offer a useful strategy to work to overcome barriers to treatment and improve engagement that may fit within the roles and responsibilities of aging service staff who recommend and link clients to multiple services. Collaboration with community providers on the design and implementation of intervention increases the likelihood that the intervention will be sustained ultimately by the community. ${ }^{54}$

\section{Acknowledgment}

This research is supported by a grant from the National Institute of Mental Health (R01 MH079265).

\section{Disclosure}

The authors have no financial conflicts to disclose in this work.

\section{References}

1. Byers AL, Yaffe K, Covinsky KE, Friedman MB, Bruce ML. High occurrence of mood and anxiety disorders among older adults: The National Comorbidity Survey Replication. Arch Gen Psychiatry. 2010;67(5):489-496.

2. Institute of Medicine of the National Academies. The mental health and substance use workforce for older adults: in whose hands? Washington, DC: National Academies Press; 2012. Available from: http://www.nap. edu/catalog.php?record_id=13400. Accessed July 25, 2013. 
3. Raue PJ, Sirey JA. Designing personalized treatment engagement interventions for depressed older adults. Psychiatr Clin North Am. 2011;34(2):489-500

4. Glanz K, Lewis FM, Rimer BK, editors. Health Behavior and Health Education: Theory, Research and Practice. San Francisco, CA: Jossey Bass; 2002.

5. Gum AM, Iser L, Petkus A. Behavioral health service utilization and preferences of older adults receiving home-based aging services. Am J Geriatr Psychiatry. 2010;18(6):491-501.

6. Charney DS, Reynolds CF 3rd, Lewis L, et al; Depression and Bipolar Support Alliance. Depression and Bipolar Support Alliance consensus statement on the unmet needs in diagnosis and treatment of mood disorders in late life. Arch Gen Psychiatry. 2003;60(7):664-672.

7. Bruce ML, Seeman TE, Merrill SS, Blazer DG. The impact of depressive symptomatology on physical disability: MacArthur Studies of Successful Aging. Am J Public Health. 1994;84(11):1796-1799.

8. Barry LC, Murphy TE, Gill TM. Depressive symptoms and functional transitions over time in older persons. Am J Geriatr Psychiatry. 2011;19(9):783-791.

9. Eggermont LH, Penninx BW, Jones RN, Leveille SG. Depressive symptoms, chronic pain, and falls in older community-dwelling adults: the MOBILIZE Boston Study. J Am Geriatr Soc. 2012;60(2): 230-237.

10. United States Department of Health and Human Services. Mental Health: A Report of the Surgeon General. Rockville, MD: United States Department of Health and Human Services, Substance Abuse and Mental Health Services Administration, Center for Mental Health Services, National Institutes of Health, National Institute of Mental Health; 1999. Available from: http://profiles.nlm.nih.gov/ps/access/ NNBBHS.pdf. Accessed July 25, 2013.

11. Abrams RC, Lachs M, McAvay G, Keohane DJ, Bruce ML. Predictors of self-neglect in community-dwelling elders. Am J Psychiatry. 2002;159:1724-1730.

12. Sheline YI. High prevalence of physical illness in a geriatric psychiatric inpatient population. Gen Hosp Psychiatry. 1990;12(6):396-400.

13. Unützer J, Patrick DL, Simon G, et al. Depressive symptoms and the cost of health services in HMO patients aged 65 years and older. A 4-year prospective study. JAMA. 1997;277(20):1618-1623.

14. Druss BG, Rohrbaugh RM, Rosenheck RA. Depressive symptoms and health costs in older medical patients. Am J Psychiatry. 1999;156(3): 477-479.

15. Luber MP, Meyers BS, Williams-Russo PG, et al. Depression and service utilization in elderly primary care patients. Am J Geriatr Psychiatry. 2001;9(2):169-176.

16. Sheeran T, Brown EL, Nassisi P, Bruce ML. Does depression predict falls among home health patients? Using a clinical-research partnership to improve the quality of geriatric care. Home Healthc Nurse. 2004;22(6):384-389

17. Blazer DG. Depression in late life: review and commentary. J Gerontol A Biol Sci Med Sci. 2003;58:249-265.

18. Unützer J, Simon G, Belin TR, Datt M, Katon W, Patrick D. Care for depression in HMO patients aged 65 and older. $J$ Am Geriatr Soc. 2000;48(8):871-878.

19. Bruce ML, Leaf PJ. Psychiatric disorders and 15-month mortality in a community sample of older adults. Am J Public Health. 1989;79(6):727-730.

20. Gallo JJ, Bogner HR, Morales KH, Post EP, Ten Have T, Bruce ML. Depression, cardiovascular disease, diabetes, and two-year mortality among older, primary-care patients. Am J Geriatr Psychiatry. 2005;13(9):748-755.

21. Bruce ML, Leaf PJ, Rozal GP, Florio L, Hoff RA. Psychiatric status and 9-year mortality data in the New Haven Epidemiologic Catchment Area Study. Am J Psychiatry. 1994;151(5):716-721.

22. Murray CJ, Vos T, Lozano R, et al. Disability-adjusted life years (DALYs) for 291 diseases and injuries in 21 regions, 1990-2010: a systematic analysis for the Global Burden of Disease Study 2010. Lancet. 2012;380(9859):2197-2223.
23. Marino P, Sirey J. Epidemiology of late life mood disorders: rates, measures and populations. In: Late Life Mood Disorders. Oxford, UK: Oxford University Press; 2012.

24. Byers AL, Arean PA, Yaffe K. Low use of mental health services among older Americans with mood and anxiety disorders. Psychiatr Serv. 2012;63(1):66-72.

25. Barry LC, Abou JJ, Simen AA, Gill TM. Under-treatment of depression in older persons. J Affect Disord. 2012;136(3):789-796.

26. Frongillo EA, Wolfe WS. Impact of participation in home-delivered meals on nutrient intake, dietary patterns, and food insecurity of older persons in New York state. J Nutr Elder. 2010;29(3):293-310.

27. Administration on Aging. Percentage Increase of the 60+ US. Population from the 2000 Census to the 2010 Census. Available from: http://www. aoa.gov/AoARoot/Aging_Statistics/Census_Population/census2010/ Index.aspx. Accessed April 25, 2012.

28. Sirey JA, Bruce ML, Carpenter M, et al. Depressive symptoms and suicidal ideation among older adults receiving home delivered meals. Int J Geriatr Psychiatry. 2008;23(12):1306-1311.

29. Richardson TM, Friedman B, Podgorski C, et al. Depression and its correlates among older adults accessing aging services. $J$ Nurs Care Qual. 2011;20(4):346-354.

30. Greenlee, K. Testimony on Senior Hunger and the Older Americans Act. 2011; Available from: http://www.help.senate.gov/imo/media/doc/ Greenlee.pdf. Accessed August 16, 2013.

31. Administration on Aging. Nutrition Program Evaluation. In: Department of Health and Human Services, editors. Serving Elders At Risk; The Older Americans Act Nutrition Programs - National Evaluation of the Elderly Nutrition Program, 1993-1995. Available from: http://www. aoa.gov/aoaroot/program_results/Nutrition_Report/eval_report.aspx. Accessed July 25, 2013.

32. Löwe B, Kroenke K, Gräfe K. Detecting and monitoring depression with a two-item questionnaire (PHQ-2). J Psychosom Res. 2005;58(2): $163-171$.

33. Spitzer R, Gibbon M, Williams JB. Structured Clinical Interview for Axis I DSM-IV Disorders (SCID). Washington, DC: American Psychiatric Association Press Inc; 1995

34. Montgomery SA, Asberg M. A new depression scale designed to be sensitive to change. Br J Psychiatry. 1979;134:382-389.

35. Raue PJ, Brown EL, Meyers BS, Schulberg HC, Bruce ML. Does every allusion to possible suicide require the same response? A structured method for assessing and managing risk. $J$ Fam Pract. 2006;55(7):605-612.

36. Bruce ML, Brown EL, Raue PJ, et al. A randomized trial of depression assessment intervention in home healthcare. $J$ Am Geriatr Soc. 2007;55(11):1793-1800.

37. Lawton MP, Brody EM. Assessment of older people: self-maintaining and instrumental activities of daily living measure. Gerontologist. 1969;9(3):179-186.

38. Folstein MF, Folstein SE, McHugh PR. "Mini-mental state". A practical method for grading the cognitive state of patients for the clinician. J Psychiatr Res. 1975;12(3):189-198.

39. Guigoz Y, Vellas B, Garry PJ. Assessing the nutritional status of the elderly: The Mini Nutritional Assessment as part of the geriatric evaluation. Nutr Rev. 1996;54(1 Pt 2):S59-S65.

40. Elliott AM, Smith BH, Penny KI, Smith WC, Chambers WA The epidemiology of chronic pain in the community. Lancet. 1999;354(9186):1248-1252.

41. Sirey JA, Meyers BS, Teresi JA, et al. The Cornell Service Index as a measure of health service use. Psychiatr Serv. 2005;56(12):1564-1569.

42. Sirey JA. Engaging to improve engagement. Psychiatr Serv. 2013; 64(3):205

43. Freeman HP. Patient navigation: a community based strategy to reduce cancer disparities. J Urban Health. 2006;83(2):139-141.

44. Jandorf L, Gutierrez Y, Lopez J, Christie J, Itzkowitz SH. Use of a patient navigator to increase colorectal cancer screening in an urban neighborhood health clinic. J Urban Health. 2005;82(2): $216-224$. 
45. Leaf PJ, Bruce ML, Tischler GL. The differential effect of attitudes on the use of mental health services. Soc Psychiatry Psychiatr Epidemiol. 1986;21(4):187-192.

46. Sirey JA, Bruce ML, Alexopoulos GS, et al. Perceived stigma as a predictor of treatment discontinuation in young and older outpatients with depression. Am J Psychiatry. 2001;158(3):479-481.

47. Sirey JA, Bruce ML, Alexopoulos GS, Perlick DA, Friedman SJ, Meyers BS. Stigma as a barrier to recovery: perceived stigma and patient-rated severity of illness as predictors of antidepressant drug adherence. Psychiatr Serv. 2001;52(12):1615-1620.

48. Miller WR, Rollnick S. Meeting in the middle: motivational interviewing and self-determination theory. Int J Behav Nutr Phys Act. 2012;9:25.

49. IBM Corp. Released 2010. IBM SPSS Statistics for Windows, Version 19.0. Armonk, NY: IBM Corp.

50. Knies KS, Marino P. The relation of depression and nutritional risk among vulnerable older adults. Presented at the 2012 Aging in America conference, Washington, DC, March 28 to April 1, 2012.

51. Richardson TM, Friedman B, Podgorski C, et al. Depression and its correlates among older adults accessing aging services. Am J Geriatr Psychiatry. 2012;20(4):346-354.
52. Colello KJ. Older Americans Act: Title III Nutrition Services Program. Washington, DC: Congressional Research Service; 2011. Available from: https://www.google.co.nz/search?q=Colello+KJ.+Older+Ameri cans+Act $\% 3 \mathrm{~A}+\mathrm{Title}+\mathrm{III}+$ Nutrition+Services+Program.+Congression al+Research+Service\%3B+2011.\&oq=Colello+KJ.+Older+American $\mathrm{s}+\mathrm{Act} \% 3 \mathrm{~A}+\mathrm{Title}+\mathrm{III}+$ Nutrition+Services+Program.+Congressional+ Research+Service\%3B+2011.\&aqs=chrome.0.69i57.1871j0\&sourcei $\mathrm{d}=$ chrome\&ie=UTF-8. Accessed July 25, 2013.

53. Substance Abuse and Mental Health Services Administration and Administration on Aging. Issue Brief 1: Aging and Behavioral Health Partnerships in the Changing Health Care Environment. Available from: http://www.aoa.gov/AoARoot/AoA_Programs/HPW/Behavioral/docs2/ Issue\%20Brief\%201\%20Partnerships.pdf. Accessed July 25, 2013.

54. Wells K, Miranda J, Bruce ML, Alegria M, Wallerstein N. Bridging community intervention and mental health services research. Am J Psychiatry. 2004;161(6):955-963.
Clinical Interventions in Aging

\section{Publish your work in this journal}

Clinical Interventions in Aging is an international, peer-reviewed journal focusing on evidence-based reports on the value or lack thereof of treatments intended to prevent or delay the onset of maladaptive correlates of aging in human beings. This journal is indexed on PubMed Central, MedLine, the American Chemical Society's 'Chemical Abstracts Ser-

\section{Dovepress}

vice' (CAS), Scopus and the Elsevier Bibliographic databases. The manuscript management system is completely online and includes a very quick and fair peer-review system, which is all easy to use. Visit http://www.dovepress.com/testimonials.php to read real quotes from published authors. 